\title{
Vereinigte Medizinische Gesellschaft in Kiew
}

\section{Sektion für Augenheilkunde}

Sitzung vom 18. Oktober 1927. Vorsitzender Prof. M. Lewitsky.

1. 0. Miroschnitschenko: Fall primärer Tuberkulose der Lidbindehaut

K. W., 30 Jahre alt. Rechtes Auge schmerzt seit einem Monat. Be-fund: Linkes Auge außer kleinen Bindehautnarben normal. Rechtes Auge: Die Lidspalte verengert; Lider verdickt, beim Betasten erscheint Tarsus etwas uneben, stellenweise findet man Erhebungen. Das ohere Lid kann man nur mit großer Mühe umstülpen. Bindehaut des oberen Lidknorpels ist infiltriert, mit Knötchen bedeckt; auch im Sulcus sub-tarsalis. Auf der Übergangsfalte fehlen die Knoten. Sie sind hirse-korngroß, gelblich, durchsichtig und erheben sich über der Bindehaut-oberfläche. Außer den Knoten findet man hier auch Bindegewebs-wucherungen und kleine Narben. Die Knoten finden sich auch auf der unteren Lidkonjunktiva. Die Hornhaut ist außen am Limbus fein getrübt. Iris, brechende Medien und Augenhintergrund ganz normal. Vis. $=0,8$. Bei mikroskopischer Untersuchung wurden typische Tuberkel mit Riesenzellen festgestellt. Wa R. negativ. Pirquet positiv. Dieser Fall gehört zur Knotenform der Bindehauttuberkulose. Auf Grund vollständigen Fehlens von tuberkulösen Erscheinungen im übrigen Körper und wegen des chronischen Krankheitsverlaufs faßt die Vor-tragende diesen Fail als einen primären tuberkulösen Prozeß auf.

2. S. Fridmann, Fall von Heterocb.romie mit einseitiger Katarakt.

K. G., 24 Jahre alt; vor einigen Jahren Schlechtersehen mit dem rechten Auge. Befund: Rechtes Auge: Lidspalte normal, Iris etwas heller als auf dem linken; Hornhaut zeigt kleine Präzipitate. Vis. $=1 / \mathrm{s}$, Projektion normal, T. $-18 \mathrm{~mm}$ hg. Beim Untersuchen mit der Spalt-lampe: Zahlreiche Präzipitate, meist flockiger Natur, teilweise mit Pig-menteinschlüssen. Regenbogenhaut ist etwas atrophisch. Am Rande der Pupille Knötchenbildungen. Linse getrübt, die vordere Embryonal-naht ist kaum zu sehen. Linkes Auge: Hypermetropie +2,0 D. Mit der Spaltlampe sind Fäden der Membrana pupillaris perseverans zu unterscheiden. Wa R. und Pirquet negativ. Der Vortragende ist der Ansicht, daß wir es hier mit einer etwas atypischen Form der Fuchs-schen Erkrankung zu tun haben. (Chronische Iridozyklitis, Katarakta complicata, Depigmentation und Atrophie der Iris.)

\section{G. Poljak. Jahresbericht über die Tätigkeit der Sektion. Die}

Sektion für Augenheilkunde zählte 192747 Mitglieder. Es haben 5 Tagungen stattgefunden, in welchen 14 Vorträge gehalten wurden. Zusammen mit der Kiewer Ophthalmologischen Gesellschaft stellte die Sektion die Zahl und Adressen aller Blinden in Kiew und Kiewer Okruge (Bezirk) fest.

I18 Vereinigie Medizinische Gesellschaft in Kiew.

In der nächsten Zeit fängt mit Hilfe aller Okulisten der Stadt eine Überschreibung dieser Blinden an, die nach der speziellen Registrie-rungskarte durchgeführt werden wird.

Nach den Vorträgen fand die Wahl des Vorstandes statt. Es wurden gewählt zum Vorsitzenden: Prof. M. Lewiisky, seine Vertreter: Priv.-Doz. /. Ginsburg und Priv.-Doz. A. Wassütinsky und zwei Sekre-täre: Dr. E. Gajewitsch und Dr. G. Poljak. 
Sitzung vom 2i. Oktober ıç2j. Vorsitzender: Prof. M. Lewiisky. i. Prof. M. Levitsky: Zum Andenken an Dr. K. Rumschewitsch.

Dr. Pinajewa: Fall doppelseitiger Kolobome der Maculae luteae.

M. Konanitzja: €ber die Topographie der Blinden im Kiewer Be-zirk. Bei der allgemeinen Überschreibung sind im Kiewer Bezirk etwa 1200 Blinde festgestellt worden, und von diesen ungefähr 300 in Kiew.

Sitzung vom 30. November IQ27. Vorsitzender: E. Gajewitsch.

1. F. Jusefowa: Fall angeborener Mißbildung der Augen. VierMonate altes Kind G. Befund: Epicantus oc. utr.

R. A. Augenbraue fehlt. Die Stirnhaut geht auf den Bulbus über und ist in der Tarsusgegend mit ihm zusammengeschmolzen. Dei Tarsus ist nicht zu fühlen. Das untere Lid besitzt den Tarsus; im äußeren Teile ist es etwas ektropioniert. Die Augenbindehaut ist nur im unteren Segmente zu sehen und ist xerotisch. Die Hornhaut ist überall, wo sie zum Vorschein kommt, trüb und xerotisch.

L. A. Die Braue ist nur an der Temporalseite entwickelt. Auf dem oberen Lid an der medialen Seite dreieckiger Defekt. Die Lidhaut ist an dieser Stelle mit dem Bulbus verwachsen. Hornhaut durchsichtig. Augenhintergrund normal.

Andere angeborene Mißbildungen: Asymmetrie des Gesichts, Sattel-gaumen, Syndactylia der Füße. Planfüße. Schwimmhäute zwischen den Fingern. Hernia umbilicalis. Geschlechtsorgane schwach entwickelt. Wa R. bei Kind und Eltern negativ. Ursache: wahrschein-lich anormale Nahtanlage.

2. M. Rutberg: Fall von Cholestearinkristallen in der Linse. KnabeW., 31/2 Jahre alt. Machte im Laufe der Zeit Keratitis, Scharlach, Keuchhusten durch. Ist etwas rachitisch.

Befund: Schon beim Tageslichte fällt Trübung seiner linken Linse ins Auge, die glitzernden, kristallischen Charakter hat. Die vordersten Schichten der Trübung sind verhältnismäßig durchsichtig, während die hinteren mehr getrübt sind. In der Dicke der Linse sind zahlreiche glänzende Kristalle von rhombischer Form, die bei Augenbewegungen ihre Stellung nicht ändern, zu unterscheiden. Außer den letzteren wird dort noch eine Menge weißer Pünktchen vorgefunden. Beim Einfall-lichte sehen sie goldgefärbt aus.

Vereinigie Medizinische Gesellschaft in Kiew. IIÇ Spaltlampenbefund: Auf der vorderen Linsenkapsel weißliche An-häufungen, im Zentrum und an der Peripherie massenhafte, durch-sichtige, glänzende Kristalle.

Aussprache: Schafran, Poljak, Gajewitsch.

3. N. Chramelow: Fall von Krönleinseher Operation bei Sehnerven-gescl $\pi v u l s t$. Die Operation wurde nach Krönlein mit Golowin scherModifikation ausgeführt. Die Geschwulst war von Taubeneigröße understreckte sich bis zum Foramen opticum. Das Endresultat war gut.

4. /. Danilewsky: Demonstration eines Talles Totischer Operation.Guter Erfolg nach der Totíschen Operation, die mit Anlegen der

Nähte zwischen Tränensack und Nasenschleimhaut ausgeführt wordenwar. G. D. Poljak. Diagnose und Therapie.

Georg H. M. Waaler (Oslo): Über die Erblichkeitsverhältnisse der ver-schiedenen Arten von angeborener Rotgrünblindheit, Acta Ophthalmo-logica Bd. V, H. 4, 1927.

G. Gekürzte Mitteilung von Studien des Verfassers

deren vollständigeDarstellung in der , Zeitschrift für induktive Abstammungs- und Vererbungslehre" erscheint. Es wird die vererbungsmäßige Bedeutung der Farben-blindheit behandelt. Zu diesem Zweck wurden sämtliche 18121 Kinderder zweiten bis achten Klasse in der Volksschule 
zu Oslo auf Farben-blindheit untersucht (9049 Knaben und 9072 Mädchen). Die Absichthierbei war eine doppelte: 1. die Familien zu finden

in denen Vererbungs-verhältnisse der Farbenblindheit in Hinsicht auf die vorliegenden Probleme genealogisch studiert werden könnten; 2. eine Übersicht überdie Häufigkeit der verschiedenen Gruppen (Protanopen

Deuteranopen, Protanomale

Deuteranomale) zu gewinnen

um Material zu einem Ver-gleich zwischen den wirklich vorkommenden Formen der

Farbenblindheit und den theoretisch erwarteten Zahlenverhältnissen zu erhalten, die Verf. in früheren Arbeiten errechnete. Als Hilfsmittel zu Massen-untersuchungen in den Schulen wurden Ishiharas pseudoisochromatischeTafeln und Nagels Anomaloskop verwendet. Das Endergebnis war:Für Mädchen ist die Häufigkeit der Farbenblindheit kaum 1/2\%. Über-wiegend sind diese deuteranomal. Für Knaben wurde etwa 1\% für jededer Gruppen: protanop deuteranop protanomal gefunden. Für dieDeuteranomalen wurden etwa $5 \%$ gefunden zusammen also 8\%. AufGrund verschiedener Erwägungen ist Verf. der Ansicht daß die Häufigkeit der Deuteranomalen etwas zu niedrig angegeben sein möchte. Erschätzt die Häufigkeit der Farbenblindheit bei Männern in Oslo auf8-9\%. Bezüglich der Einzelheiten des wesentlichen Inhalts der hoch-interessanten Arbeit insbesondere der Beziehungen die sich dem Verf.zur Chromosomenhypothese und zu anderen Fragen neuester Erblichkeitsprobleme ergaben

muß auf die Originalarbeit verwiesen werden, deren Erscheinen hier nur angezeigt werden sollte. Junius

Bonn. 